\title{
ANÁLISIS DE LA RELACIÓN ENTRE EL VALOR BURSÁTIL / VALOR CONTABLE Y LA APLICACIÓN DE LA NORMA CONTABLE (Por deterioro de Activos)
}

\author{
ANALYSIS OF THE RELATIONSHIP BETWEEN THE MARKET VALUE / BOOK VALUE \\ AND APPLICATION OF ACCOUNTING STANDARDS (IMPAIRMENT OF ASSETS)
}

\author{
Percy Antonio Vilchez Olivares \\ Docente Asociado de la Facultad de Ciencias Contables - UNMSM \\ [Recepción: Marzo de 2011/ Conformidad: Abril de 2011]
}

\section{RESUMEN}

El presente artículo presenta un análisis de la métrica Valor Bursátil/ Valor Contable de las empresas que cotizan en la Bolsa de Valores de Lima, y como esta relación es considerada por la Norma Internacional de Contabilidad Deterioro del Valor de los Activos en el proceso de evaluar indicios de deterioro por parte de la empresa. $\mathrm{Al}$ respecto, en el artículo se describe los aspectos conceptuales y normativos de la Norma Deterioro del Valor de los Activos, la Metodología para la aplicación del Deterioro del Valor de los Activos, un análisis de los resultados de la relación Valor Bursátil/ Valor Contable que presentan las empresas que cotizan en Bolsa de Valores de Lima, y su impacto en el establecimiento de indicios de deterioro en las empresas.

Palabras Clave: Deterioro de activos, valor bursátil/valor contable, Norma contable.

\section{INTRODUCCIÓN}

La globalización de los negocios y los mercados ha generado la necesidad de contar con estándares de información financiera de uso global, en ese sentido, los organismos reguladores de las Bolsas de Valores del Mundo hace una década han decidido recomendar la aplicación de estándares contables interna-

\begin{abstract}
This article presents an analysis of the metric Market Value / Book Value of companies listed on the Lima Stock Exchange, and as this relationship is considered by the International Accounting Standard for Impairment of Assets in the process of evaluating signs of deterioration by the company.

In this regard, the article describes the conceptual and normative aspects of the Standard Impairment of Assets, Methodology for the implementation of Impairment of Assets, an analysis of the results of the relation Market Value / Book Value to submitted by companies listed on the Lima Stock Exchange, and its impact on establishing evidence of deterioration in the business.
\end{abstract}

Keywords: Impairment of assets, market value / book value, accounting standard.

\footnotetext{
* Magíster en Ciencias contables y financieras - Universidad Diego Portales - CHILE. Docente Investigador de la Facultad de Ciencias Contables - UNMSM. E- mail: pvilchez20@hotmail.com
}

cionales en la preparación de estados financieros de las empresas que cotizan en las Bolsas de Valores del Mundo. La aplicación de estándares contables permite mayor calidad y mayor transparencia, características que se encuentran establecidas en las Normas Internacionales de Información Financiera. 
En nuestro país la Comisión Nacional Supervisora de Valores CONASEV es la entidad encargada de establecer la normatividad contable y los reglamentos de preparación de información financiera para las empresas cotizadas en la Bolsa de Valores de Lima.

$\mathrm{Al}$ respecto, este organismo regulador contable ha emitido la Res. de CONASEV No 102-2010-EF/94.01 que establece que las sociedades emisoras de valores inscritos en el registro público del mercado de valores deben preparar sus estados financieros con observancia a las Normas Internacionales de Información Financiera NIIF, con lo cual en nuestra país se inicia el proceso de adopción plena de las NIIF a partir de los estados financieros auditados al 31 de diciembre del 2011.

Este proceso de adopción plena implica una revisión y aplicación exhaustiva de la normatividad internacional, siendo la Norma Internacional de Contabilidad NIC 36 Deterioro del Valor de los Activos una norma que requiere un trabajo muy exhaustivo en el proceso de su implementación y cumplimiento, y también una norma que requiere conocer los resultados financieras de las empresas en el entorno, y como esta tienen un impacto en el proceso de la adopción plena de la mormativa.

\section{MARCO LEGAL DE APLICACIÓN DE NIIF EN PERÙ}

El Comité de Normas Internacionales de Contabilidad (IASC, por sus siglas en inglés) fue creado en 1973, entidad que emite las Normas Internacionales de Contabilidad. Su creación está vinculada con la designación de la Federación Internacional de Contadores, de emitir estándares contables internacionales. IFAC es la organización mundial que representa a las instituciones de contabilidad en el mundo, siendo su objetivo principal desarrollar Estándares Contables para la profesión y promover su aceptación y adopción internacional.

En el año 2001, el Comité de Normas Internacionales de Contabilidad se disolvió y se creó la Junta de Normas Internacionales de Contabilidad, (IASB, por sus siglas en inglés) asumiendo la responsabilidad para regular la presentación de estándares contables y financieros a nivel global. Asimismo, se renombró las Normas Internacionales de Contabilidad a Normas Internacionales de Información Financiera.

En nuestro país, con la vigencia Ley General de Sociedades Ley No 26887 de 1997 ,se establece que los estados financieros de las empresas deben ser preparados y presentados de acuerdo a disposiciones legales y Principios de Contabilidad Generalmente Aceptados; al respecto, el Consejo Normativo de Contabilidad ha precisado, a través de la Resolución No 013-98-EF/93.01, que los Principios de Contabilidad Generalmente Aceptados a los que se hace referencia son las Normas Internacionales de Información Financiera (antes Normas Internacionales de Contabilidad). Asimismo, el Consejo Normativo de Contabilidad órgano de la Contaduría Pública de la Nación es la entidad encargada de oficializar y difundir la normativa internacional.

Asimismo, de acuerdo al Texto Único Ordenado de la Ley del Mercado de Valores, aprobado con Decreto Supremo No 0932002-EF, corresponde a la Comisión Nacional Supervisora de Empresas y Valores CONASEV establecer la normatividad contable para la elaboración de los estados financieros de las entidades sometidas a su control y supervisión, así como la forma de presentación de dichos estados financieros. 
La Resolución CONASEV No 0922005-EF/94.10 dispuso que a partir del 01 de enero del 2006, las empresas sujetas al control y supervisión de CONASEV deberan sujetarse a lo dispuesto a las Normas Internacionales de Información Financiera.

Para cumplir con el proceso de adopción de las Normas Internacionales de Información Financiera se ha emitido la Resolución CONASEV No102-2010-EF/94 01.1 mediante la cual se establece que las empresas emisoras de valores inscritos en el Registro Público del Mercado de Valores, las empresas clasificadoras de riesgo, entre otras, deben preparar sus estados financieros con observancia plena de las Normas Internacionales de Información Financiera (NIIF), que emita el IASB vigente internacionalmente.

De acuerdo con dichas normas, los primeros estados financieros que deben formular las empresas aplicando plenamente las NIIF serán los correspondientes a la información financiera auditada anual al 31 de diciembre del 2011 y los posteriores estados financieros trimestrales correspondientes al 2012.

\section{NORMA CONTABLE SOBRE DETE- RIORO DEL VALOR DE LOS ACTIVOS}

En nuestro país la aplicación de la NIC 36 Deterioro del Valor de los Activos (antes Desvalorización de Activos) ha sido oficializada por el Consejo Normativo de Contabilidad mediante Resolución No 0342005-EF/93.01 del 17 de febrero del 2005 publicada el 02 de marzo del 2005 siendo la aplicación obligatoria para los estados financieros de los ejercicios económicos que comienzan el 01 de enero del 2006.

La Junta de Normas de Contabilidad Financiera (IASB, por sus siglas en inglés) llevo a cabo la revisión de la NIC 36 Deterioro del Valor de los Activos como consecuencia de la revisión de la NIC 1 Presentación de Estados Financieros (revisada en 2007) y la NIIF 3 Combinación de Negocios (revisada en 2008). Una entidad aplicará esas modificaciones para los periodos anuales que comiencen a partir del 1 de julio de 2009. Estas modificaciones tienen vigencia en nuestro país mediante Resolución emitida por Contaduría Pública de la Nación Resolución No 044-2010-EF/ 94 publicada el 28 de agosto del 2010 siendo la aplicación obligatoria para los estados financieros de los ejercicios económicos que comienzan el 01 de enero del 2011.

Asimismo, para las empresas que cotizan en Bolsa de Valores de Lima, se ha emitido la Resolución CONASEV No102-2010-EF/94 01.1 sobre aplicación plena de las NIF , para los estados financieros emitidos al 31 de diciembre del 2011, en consecuencia, las empresas peruanas que cotizan en Bolsa de Valores deberán aplicar la NIC 36 Deterioro del Valor de los Activos, que tiene por objetivo establecer los procedimientos que una empresa aplicará para asegurarse de que sus activos están contabilizados por un importe que no sea superior a su importe recuperable. Un activo estará contabilizado por encima de su importe recuperable cuando su importe en libros exceda del importe que se pueda recuperar del mismo a través de su utilización o de su venta. Si este fuera el caso, el activo se presentaría como deteriorado, y la Norma exige que la empresa reconozca una pérdida por deterioro del valor de ese activo. En la Norma también se especifica cuándo la empresa revertirá la pérdida por deterioro del valor, así como la información a revelar.

Es importante precisar que la norma ha definido como activos sujetos de deterioro a Propiedades, Plantas y Equipos, Activos intangibles, Plusvalías Compradas, Inversiones 
Inmobiliarias Valoradas y los activos que no poseen mecanismos de deterioro en sus propias Normas Internacionales de Información Financiera.

\section{METODOLOGÍA EN LA DETERMI- NACIÓN DE DETERIORO DE ACTI- VOS}

Para efectos de reconocer el Deterioro del Valor de los Activos, se debe seguir diversas acciones como parte integrante de un proceso secuencial. Los procesos que se pueden delimitar son los siguientes:

a) Identificación de Activos Sujetos a Deterioro, para efectos de identificar activos sujetos a deterioro se analizara el contenido de los activos que integran el Estado de Situación Financiera, con la consecuencia evaluación de su materialidad.

b) Evaluación de Indicios de Deterioro, para determinar si un activo esta deteriorado, se considerara como mínimo la evaluación de los indicios de deterioro. La NIC 36 en sus párrafos 12 al 14 describe algunos indicadores para comprobar si existe una pérdida por deterioro del valor de un activo. Si se cumpliese alguno de esos indicadores, la entidad estará obligada a realizar una estimación formal del valor recuperable realizando un test de deterioro.

c) Determinación del Valor Recuperable, la determinación del valor recuperable del activo se efectuará sobre la base del mayor valor de comparar el valor razonable menos los costos de ventas del activo y su valor de uso del activo. En caso de no poder determinar el valor razonable menos los costos de ventas del activo porque no hay base para estimar un valor confiable se optará por el valor de uso del activo como su valor recuperable.
El valor razonable menos los costos de ventas se determinará mediante evidencia en el siguiente orden: precios de venta corriente, el precio de la transacción más reciente menos los costos de disposición del activo. El valor de uso se determinará considerando los flujos de efectivo futuros estimados que la entidad espera derivar del activo, las expectativas sobre los cambios en las cantidades o en la oportunidad de estos flujos de efectivo futuros afectándolo por la tasa de descuento que refleje el ajuste al valor del dinero en el tiempo. En la determinación de los flujos de efectivos futuros para cada unidad generadora de efectivo las proyecciones cubrirán períodos no mayores a cinco años, a menos que se justifique un período más largo y esas estimaciones son confiables.

d) Comparación del Valor Recuperable con el Valor en Libros, para efectos de comparar se procederá a comparar ambos valores, si el valor recuperable del activo es menor que su valor en libros, la empresa debe reducir el valor en libros de su activo hasta alcanzar su valor recuperable, generando una pérdida por deterioro en los registros contables y el reconocimiento del impuesto a la renta diferido respectivo. La disminución del valor en libros del activo deteriorado debe ser reconocida afectando los resultados del período si la empresa contabiliza dicho activo bajo el método del costo o debe ser cargado a la cuenta patrimonial excedente de revaluación si se contabiliza el activo a valores revaluados.

\section{EVALUACIÓN DE LOS INDICIOS DE DETERIORO}

Para efectos de cumplir con la metodología señalada b) Evaluación de Indicios de 
Deterioro, se deberá aplicar la Norma Internacional de Contabilidad NIC 36 párrafo 12, en el que se precisa que al evaluar si existe algún indicio de que el valor del activo puede haberse deteriorado, la entidad considerará, como mínimo, las siguientes fuentes de información:

\section{Fuentes externas de información}

(a) Durante el periodo, el valor de mercado del activo ha disminuido significativamente más que lo que cabría esperar como consecuencia del paso del tiempo o de su uso normal.

(b) Durante el periodo han tenido lugar, o van a tener lugar en un futuro inmediato, cambios significativos con una incidencia adversa sobre la entidad, referentes al entorno legal, económico, tecnológico o de mercado en los que ésta opera, o bien en el mercado al que está destinado el activo.

(c) Durante el periodo, las tasas de interés de mercado, $u$ otras tasas de mercado de rendimiento de inversiones, han sufrido incrementos que probablemente afecten a la tasa de descuento utilizada para calcular el valor en uso del activo, de forma que disminuyan su importe recuperable de forma significativa.

(d) El importe en libros de los activos netos de la entidad, es mayor que su capitalización bursátil.

\section{Fuentes internas de información}

(e) Se dispone de evidencia sobre la obsolescencia o deterioro físico de un activo.

(f) Durante el periodo han tenido lugar, o se espera que tengan lugar en un futuro inmediato, cambios significativos en el alcance o manera en que se usa o se espera usar el activo, que afectarán desfavorable- mente a la entidad. Estos cambios incluyen el hecho de que el activo esté ocioso, planes de discontinuación o restructuración de la operación a la que pertenece el activo, planes de vender o disposición por otra vía del activo antes de la fecha prevista, y la reconsideración como finita de la vida útil de un activo como finita, en lugar de indefinida.

(g) Se dispone de evidencia procedente de informes internos, que indica que el rendimiento económico del activo es, o va a ser, peor que el esperado.

De acuerdo a la Norma Internacional de Contabilidad NIC 36 párrafo 17 se señala que "Si existiese algún indicio de que el activo puede haber deteriorado su valor, esto podría indicar que, la vida útil restante, el método de depreciación (amortización) o el valor residual del activo, necesitan ser revisados y ajustados de acuerdo con la Norma aplicable a ese activo, incluso si finalmente no se reconociese ningún deterioro del valor para el activo considerado".

Dado este enunciado, podría no estar reconociéndose en los estados financieros de la empresa una provisión por deterioro por la existencia de algún indicio relacionado a sus activos.

\section{MÉTRICA VALOR BURSÁTIL/VALOR CONTABLE}

Considerando que uno de los cuatro indicios externos en el párrafo 12 de la Norma Internacional de Contabilidad NIC 36 se refiere al tema de la relación Valor Bursátil/ Valor Contable, específicamente en d) "El importe en libros de los activos netos de la entidad, es mayor que su capitalización bursátil”.

$\mathrm{Al}$ respecto, la métrica Valor Bursátil/Valor Contable muestra la relación de veces en- 
tre el Valor Bursátil (El Patrimonio Bursátil es la cantidad de acciones corrientes suscritas y pagadas por su precio) y el Valor Contable (El Patrimonio Contable es la cantidad de acciones suscritas y pagadas multiplicadas por el valor contable de la acción)

En ese sentido, es necesario analizar los resultados de las empresas que cotizan en la Bolsa de Valores de Lima, para efectos de evaluar el cumplimiento del indicio de deterioro.

En el Cuadro No 1 se presenta el análisis del Reporte Diario de Mercados al 01 de febrero del 2011 de las acciones cotizadas en la Bolsa de Valores de Lima.

Tal como se puede apreciar en el Cuadro No 1, las empresas agroindustriales son las que están más expuestas al cumplimiento en el estado de indicio de deterioro, como consecuencia de ser un sector industrial con bajos rendimientos financieros y no muy atractivos los flujos de efectivos futuros. Al respecto una Empresa Minera, Morococha tiene un Ratio M/B 0.9X y dos Empresas Agroindustriales, la empresa Pomalca tiene un Ratio M/B 0.6X y la empresa Tuman tiene un Ratio $\mathrm{M} / \mathrm{B} 0.5 \mathrm{X}$, estos resultados son significativos para efectos establecer que existen indicios de deterioro.

Claro esta, que debemos identificar en sus estados financieros la cuantia de los activos contables sujetos a deteriorar en relación a su peso respecto al activo total.

El Cuadro No 2 se presenta para las tres empresas que presentan significativo indicio de deterioro la relación Valor Bursátil/Valor Contable y el resultado obtenido de la relación Activos Susceptibles de Deterioro/Activo Total sobre estados financieros al $31 \mathrm{de}$ diciembre del 2009, al no estar disponibles a los stakeholders los estados financieros al 31 de diciembre del 2010.

Si bien es cierto, el Cuadro No 2 Presenta un ratio de Activos Susceptibles de Deterio-

Cuadro No 1. Empresas Cotizadas en Bolsa de Valores de Lima al 01/02/2011

\begin{tabular}{|l|c|c|c|c|}
\hline $\begin{array}{l}\text { Acciones } \\
\text { Sector Industrial }\end{array}$ & $\begin{array}{l}\text { Número de } \\
\text { Empresas }\end{array}$ & $\begin{array}{l}\text { Promedio del Sector } \\
\text { en el Ratio M/B }\end{array}$ & $\begin{array}{l}\text { Núm. de Empresas con } \\
\text { Ratio M/B Menor a 1 }\end{array}$ & $\begin{array}{l}\text { Núm. de Empresas con } \\
\text { Ratio M/B Mayor a 1 }\end{array}$ \\
\hline Energía & 5 & $3.7 \mathrm{X}$ & 0 & 5 \\
\hline Minería & 13 & $8.3 \mathrm{X}$ & 1 & 5 \\
\hline Bancos & 5 & $3.9 \mathrm{X}$ & 0 & 12 \\
\hline Construcción & 10 & $3.5 \mathrm{X}$ & 0 & 2 \\
\hline Industriales & 2 & $3.0 \mathrm{X}$ & 0 & 4 \\
\hline Agroindustriales & 6 & $1.4 \mathrm{X}$ & 2 & \\
\hline
\end{tabular}

Fuente: Reporte Diario de Mercados BBVA Research.Bloomberg,Economática

Notas: M / B Market - Book : Ratio Valor Bursátil/ Valor Contable

Cuadro No 2. Empresas con relación Valor Bursátil/Valor Contable menor a 1 y el Nivel de Activos Susceptibles de Deterioro al 31/12/2009

\begin{tabular}{|l|c|c|}
\hline \multicolumn{1}{|c|}{ Empresa } & Ratio M/B & Ratio ASD/AT \\
\hline Morococha S.A. & $0.9 \mathrm{X}$ & 0.86 \\
\hline Pomalca & $0.6 \mathrm{X}$ & 0.90 \\
\hline Tuman & $0.5 \mathrm{X}$ & 0.88 \\
\hline
\end{tabular}

Fuente: Elaboración Propia

Notas: ASD/AT Ratio Activos Susceptibles de Deterioro/Activo Total 
ro versus el Activo total sobre cifras al 31 de diciembre del 2009, estos resultados deben ser corroborados en su tendencia al 31 de diciembre del 2010 y periódos subsecuentes para establecer que parte significativa del patrimonio de la entidad esta sujeta a los alcances de la Norma Internacional de Contabilidad NIC 36 Deterioro de Activos.

Respecto a los resultados presentados en las tres empresas, estas presentan una relevancia en el ratio de Activos Susceptibles de Deterioro versus el Activo Total mayor al 0.5 lo cual indica la significancia que puede tener en los estados financieros el reconocimiento del Deterioro de Activos en las empresas analizadas.

\section{CONCLUSIONES}

1. La gran mayoría de las empresas que cotizan sus acciones en la Bolsa de Valores de Lima no estará afecta a cumplir con uno de los indicios de deterioro señalados en la NIC 36 sobre "que el valor en libros es mayor a su valor bursátil”, por lo tanto, no se reconocerá perdidas por deterioro en los estados financieros de estas empresas.

2. La Norma Internacional de Contabilidad define un proceso para establecer reco- nocimientos de perdidas por deterioro de activos sujeto a factores externos e internos señalados en los indicios de deterioro, y que consecuentemente recoge las expectativas de los inversionistas por nuestra empresa traducido en el valor bursátil de la acción.

\section{REFERENCIAS BIBLIOGRÁFICAS}

1. Alexander David, Y Archer Simon (2005). Guía Miller de NIC/ NIIF. Editorial CCH Incorporated - Perú Federación de Colegios de Contadores Públicos del Perú (2000). "Normas Internacionales de Contabilidad”. Impreso en Perú.

2. International Accounting Standards Committee Foundation IASCF (2001). Marco Conceptual para la Preparación de Estados Financieros.

3. Jara Sarrúa (2008). Relación Bolsa/Libro y su incidencia en la aplicación de la NIC 36.

4. Revista contabilidad y sistemas. Universidad de Chile - CHILE

5. IASB http://www.iasb.org.uk (visitado El 08 de febrero- 2011)

6. IFAC http://www.ifac.org/.(visitado El 08 de febrero- 2011) 